\title{
Comparative Effectiveness of Adjuvant Chemotherapy in Elderly Colon Cancer Patients
}

\author{
Sacha Satram-Hoang ${ }^{1}$, Luen Lee ${ }^{2}$, Shui $\mathrm{Yu}^{2}$, Faiyaz Momin ${ }^{1}$, \\ Sridhar R. Guduru ${ }^{1}$, Carolina Reyes ${ }^{2} \&$ Edward McKenna ${ }^{2}$ \\ ${ }^{1}$ Q.D. Research, Inc., Granite Bay, USA \\ ${ }^{2}$ Genentech, Inc., 1 DNA Way, South San Francisco, USA \\ Correspondence: Sacha Satram-Hoang, Ph.D., President, Q.D. Research, Inc. 8789 Auburn Folsom Road C501, \\ Granite Bay, CA 95746, USA. Tel: 1-949-413-5032. Fax: 1-916-865-4162. E-mail: sacha@qdresearch.com
}

Received: Januray 18, 2013 Accepted: February 3, 2013 Online Published: February 20, 2013

doi:10.5539/cco.v2n1p115 URL: http://dx.doi.org/10.5539/cco.v2n1p115

\begin{abstract}
Introduction: We assessed whether older patients experience the same treatment benefits from adjuvant chemotherapy in a real-world setting as younger patients in clinical trials. Methods: This retrospective cohort analysis used the linked Surveillance, Epidemiology, and End Results (SEER)-Medicare database. The analysis included 3,390 patients, $>65$ years, diagnosed with stage II or III colon cancer between January 1, 2004, and December 31, 2007. Patients were enrolled in Medicare Parts A and B, and had undergone adjuvant treatment with fluorouracil plus leucovorin (5-FU/LV; $n=1,368$ ), 5-FU/LV plus oxaliplatin (FOLFOX; $n=1,398$ ), capecitabine (CAP; $n=507)$, or CAP plus oxaliplatin (CAPOX; $n=117$ ) within 3 months of surgery. Differences in patient demographics and disease characteristics by treatment were assessed by chi-square test and ANOVA or t-test. Cox proportional hazards regression and propensity score-weighted analyses were used to measure the relative risk of death associated with each treatment. Results: Median time from surgical resection to initiation of treatment was similar across cohorts (44-48 days). Mean treatment duration was longer for 5-FU/LV (149 days) and FOLFOX (144 days) than CAP (121 days) and CAPOX (111 days); $\mathrm{p}<.0001$. In the propensity score-adjusted survival models, patients treated with FOLFOX (HR $=0.70 ; 95 \% \mathrm{CI}=0.55-0.90)$ and $\mathrm{CAPOX}(\mathrm{HR}=0.44 ; 95 \% \mathrm{CI}=0.20-0.98)$ had a significantly lower risk of death than $5 \mathrm{FU} / \mathrm{LV}$, while risk of death was similar between CAP and 5-FU/LV. Conclusions: Elderly patients with colon cancer have similar benefits from adjuvant chemotherapies in real-world settings as demonstrated in younger patients in clinical trial settings.
\end{abstract}

Keywords: colon cancer, adjuvant, chemotherapy, survival, elderly

\section{Introduction}

Surgical resection is the only curative treatment for locoregional colon cancer. Studies have shown that adjuvant chemotherapy reduces risk of relapse and prolongs survival in Stage III (node-positive) colon cancer (Investigators, 1995; Moertel et al., 1995; Wolmark et al., 1993), although the benefits of adjuvant chemotherapy in Stage II (node-negative) disease are less certain. Bolus fluorouracil plus leucovorin (5-FU/LV) has been the standard of care for colon cancer adjuvant treatment since the early 1990s. The oral fluoropyrimidine capecitabine (CAP) is an effective alternative to intravenous $5-\mathrm{FU} / \mathrm{LV}$ in the adjuvant setting and has demonstrated equivalent disease-free survival (DFS) and overall survival (OS) with significantly fewer adverse events (AEs) (Scheithauer et al., 2003; Twelves et al., 2011; Twelves et al., 2005). In addition, capecitabine plus oxaliplatin (CAPOX) and 5-FU/LV plus oxaliplatin (FOLFOX) have demonstrated significantly better DFS and OS than bolus 5-FU/LV (Andre et al., 2004; Andre et al., 2009; Haller et al., 2011; Kuebler et al., 2007; H.-J. Schmoll et al., 2012).

Although almost three-quarters of patients with colon cancer are 65 years or older; clinicians are less likely to follow expert recommendations for adjuvant chemotherapy with older patients than with younger patients (Sundararajan et al., 2002; Wolpin, Meyerhardt, Mamon, \& Mayer, 2007). A recent analysis of data on more than 85,000 patients with colon cancer found that only $69 \%$ of patients aged $70-79$ years and $39 \%$ of those aged 80 or older received adjuvant chemotherapy, compared to $82 \%$ of patients younger than 60 , and $77 \%$ of patients aged 60-69 years (Jessup, Stewart, Greene, \& Minsky, 2005). Comorbid health conditions and older age appear to influence physicians' choice of treatment regimen due to tolerability issues and discourage administration of recommended therapies (Kahn et al., 2010; O'Grady et al., 2011; Schrag, Cramer, Bach, \& Begg, 2001). 
Elderly patients have been historically underrepresented in clinical trials, providing a limited evidence base in which to evaluate the benefits of adjuvant chemotherapy in this population. Furthermore, data on real-world outcomes with newer alternative agents are rare. The National Comprehensive Cancer Network (NCCN) recommends that patients with high-risk Stage II or Stage III colon cancer receive adjuvant chemotherapy with FOLFOX, bolus 5-FU/LV plus oxaliplatin, or CAPOX; and single-agent CAP or 5-FU-LV for patients inappropriate for oxaliplatin therapy. Fluoropyrmidine monotherapy, either CAP or 5-FU/LV, may be considered for patients with low-risk Stage II colon cancer (National Comprehensive Cancer Network, 2012). Per these guidelines, and the need for comparative effectiveness research in this area, we evaluated Stage II and Stage III colon cancer patients receiving adjuvant chemotherapy with 5FU/LV, CAP, CAPOX and FOLFOX in a real-world setting. With the increasing size of the elderly population in the United States, the results of this study may provide an important and timely context for identifying opportunities to improve the quality of colon cancer treatment strategies and provide a better understanding of the clinical benefits of adjuvant chemotherapy in the real-world setting compared to younger clinical trial participants.

\section{Methods}

\subsection{Data Sources}

We used population-based claims data from the Surveillance, Epidemiology, and End Results (SEER)-Medicare linked database. Institutional review board approval was waived because the SEER-Medicare data lack personal identifiers. The SEER-Medicare database is a collaborative effort of the National Cancer Institute (NCI), SEER registries, and Centers for Medicare and Medicaid Services (CMS). The SEER-Medicare database provides information on Medicare patients included in SEER, a nationally representative collection of 18 population-based registries of all incident cancers from diverse geographic areas covering approximately $28 \%$ of the U.S. (Warren, Klabunde, Schrag, Bach, \& Riley, 2002). All incident cancer patients reported to the SEER registries are cross-matched with a master file of Medicare enrollees (Potosky, Riley, Lubitz, Mentnech, \& Kessler, 1993). Approximately $97 \%$ of U.S. residents aged 65 years or older are eligible for Medicare and all Medicare beneficiaries receive Part A coverage (inpatient care, skilled nursing, home healthcare, and hospice care) while approximately $95 \%$ of beneficiaries subscribe to Part B, which covers physician services and outpatient care. The current analysis included all Medicare-eligible persons with data in SEER through 2007 and their Medicare claims for Part A and Part B through 2009.

\subsection{Study Population}

Patients were included in the study if they were diagnosed with a first primary Stage II or Stage III colon cancer from January 1, 2004 to December 31, 2007, were at least 66 years of age, had undergone resection of the primary tumor within 3 months after diagnosis, and received adjuvant chemotherapy within 3 months after resection. Consistent with prior studies of adjuvant chemotherapy use, we excluded patients who died within 6 months of surgery to restrict the sample to patients who did not have a poor short-term prognosis that would disqualify them for consideration of adjuvant therapy (Gross, McAvay, Guo, \& Tinetti, 2007; Schrag, Cramer, et al., 2001). Patients were excluded if their date of death was recorded prior to or the same month as diagnosis, Medicare Parts $\mathrm{A}$ and $\mathrm{B}$ enrollment was less than 12 months prior to diagnosis date, enrolled in a health maintenance organization (HMO) during any period of the 12 months prior to diagnosis (because data were unavailable for these periods) and had two or more claims for chemotherapy prior to diagnosis. Of the 4593 patients who met all study criteria, 1368 received adjuvant treatment with 5FU/LV, 1398 received FOLFOX, 507 received CAP, and 117 received CAPOX.

\subsection{Study Variables}

The SEER program registries routinely collect data on patient demographics (age, race/ethnicity, residence, socioeconomic status [income and education per census tract]), primary tumor site, tumor morphology, stage at diagnosis, first course of treatment, and follow-up for vital status. Median annual household income at the census tract level and percentage of the adult population who completed specific levels of education at the ZIP code level were used as a proxy for socioeconomic status. SEER site codes were used to identify colon. We considered both the American Joint Committee on Cancer (AJCC) and SEER stage groupings to identify the stage at diagnosis.

The SEER program does not record chemotherapy administration. Data was abstracted from four merged SEER-Medicare files: Medicare provider analysis and review (MEDPAR), carrier claims $(\mathrm{NCH})$, outpatient claims (OUTSAF) and durable medical equipment (DME) to identify claims for chemotherapy administration (Warren, Harlan, et al., 2002). Claims for oral equivalents of IV chemotherapies were identified in the DME file (i.e. capecitabine). Chemotherapy were characterized and quantified using International Classification of Disease (ICD) diagnosis codes, ICD procedural codes, Current Procedural Terminology (CPT) codes, Healthcare Common 
Procedural Coding System (HCPCS) codes and revenue center codes. Chemotherapy claims were searched for specific drug codes to identify the type of chemotherapy used. The absence of these claims indicated lack of treatment. The first chemotherapy claim within 3 months after surgical resection indicated the start of adjuvant therapy. Patients were classified into one of four treatment groups (5FU/LV, CAP, FOLFOX, CAPOX) based on all chemotherapy given to a patient during the first 60 days after date of initiation of treatment. Medicare claims were used to identify patients who underwent primary resection of the tumor within 3 months after diagnosis. Colon cancer surgical procedures included hemicolectomy, subtotal colectomy, and total colectomy.

We aggregated comorbidities to formulate the NCI comorbidity index, a revised version of the Charlson comorbidity index. (Klabunde, Legler, Warren, Baldwin, \& Schrag, 2007) Complications from adjuvant treatment within 180 days after treatment initiation were identified by ICD-9 diagnosis codes in both inpatient and outpatient Medicare claims. This 180-day period was selected based on the National Comprehensive Cancer Network (NCCN) guidelines that recommend 6 months of adjuvant treatment for stage II and III CRC or for stage IV stable disease (NCCN, 2012).

\subsection{Statistical Analysis}

Demographic and clinical characteristics were summarized descriptively by treatment type. Treatment patterns delivered in the adjuvant setting included: treatment type by time of initiation from surgery, duration of treatment, and complications of therapy within 180 days from treatment initiation. Chi-square test for categorical variables and ANOVA or t-test for continuous variables determined differences between groups. We considered a $p$-value $<.05$ to be statistically significant.

We compared two approaches as a sensitivity exercise to assess overall risk of death: (1) Cox proportional hazards regression and (2) propensity score-weighted Cox proportional hazards regression model. In the Cox proportional hazards regression, we adjusted the multivariate models for confounders that were selected from demographic and clinical characteristics using the backward elimination strategy-a step-wise removal of covariates in full the model until only the significant variables remain (Greenland, 1998). In the second approach, multinomial logistic regression was used to calculate a propensity score - the conditional probability that each patient would receive a specific treatment given that patient's pretreatment variables (baseline characteristics, such as age, gender, and comorbidities) - for each patient (Kurth et al., 2006). We fitted the propensity score-weighted Cox proportional hazards regression model to compare overall survival in the four treatment groups. Follow-up was calculated beginning on the date of treatment initiation up until the first occurrence of a censoring event: date of death, development of a second primary tumor, the last date for which Medicare claims are available, or the end of the follow-up period (December 31, 2009). All statistical analyses were performed using SAS software, version 9.1.3 (SAS Institute Inc., Cary, North Carolina).

\section{Results}

\subsection{Demographic \& Clinical Characteristics}

The mean age at diagnosis was 73 in the FOLFOX group, 74 for CAPOX, 75 for 5-FU/LV, and 77 for CAP $(\mathrm{p}<0.0001)$. In general, patients treated with CAP were older, more likely to be female and non-white, and had a higher comorbidity score than patients in the other treatment groups (Table 1). A higher proportion of CAPOX patients were from the western part of the United States, and they had higher income and education levels than the other treatment groups. 
Table 1. Demographic and Clinical Characteristics at Baseline

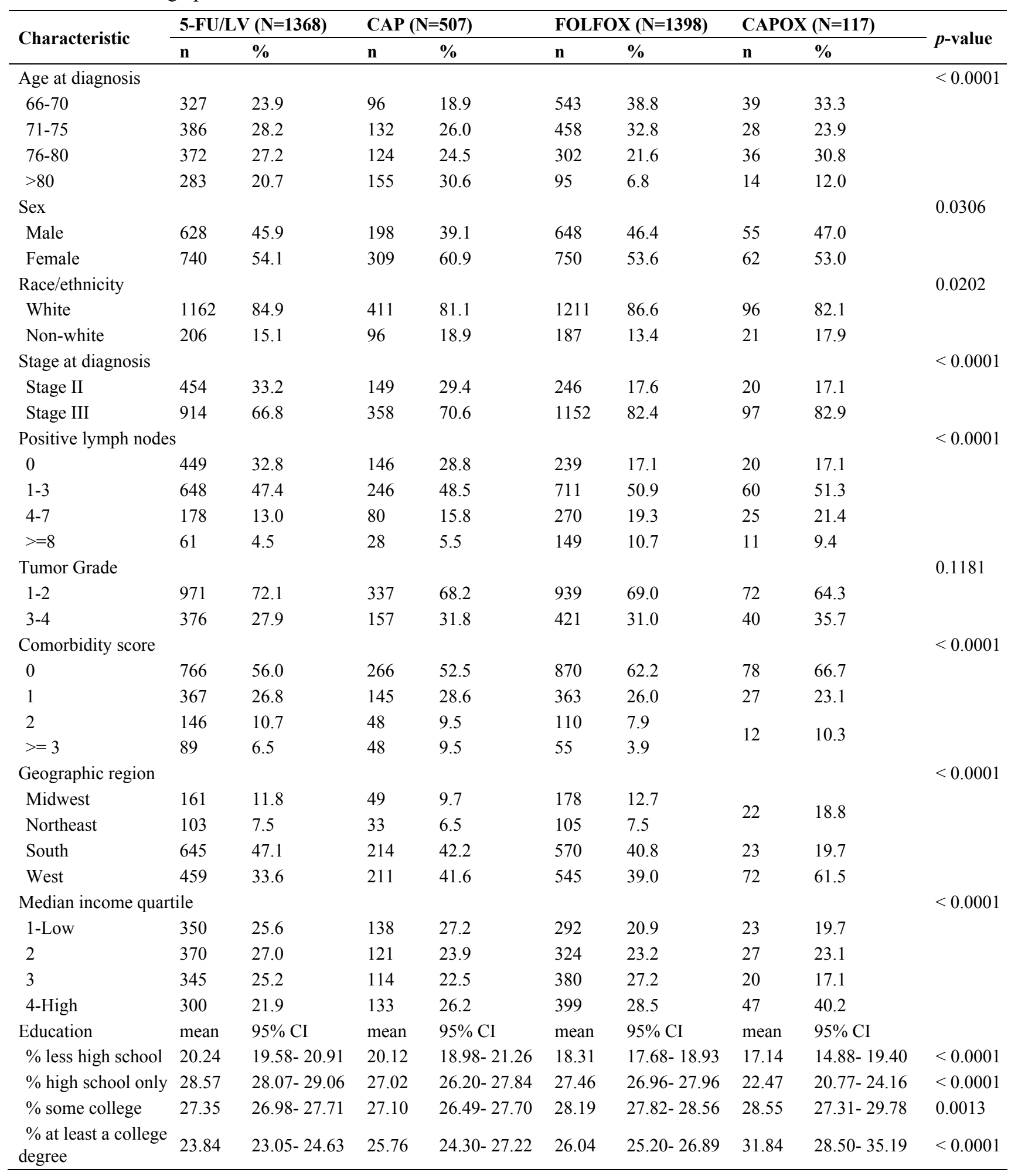

Note: Cells with counts of less than 11 are combined in compliance with the National Cancer Institute data use agreement for small cell sizes.

\subsection{Treatment Patterns}

Throughout the study time period, the use of CAP (6\% to 23\%) and FOLFOX (20\% to 57\%) increased from 2004 to 2009 , while the use of 5-FU/LV (72\% to 19\%) decreased (Figure 1). The mean time (46-49 days) to chemotherapy initiation after surgery was similar between treatment groups (Table 2). Duration of treatment was 
longer for 5-FU/LV (149 days) and FOLFOX (144 days) than for CAP (121 days) and CAPOX (111 days; Table 2).

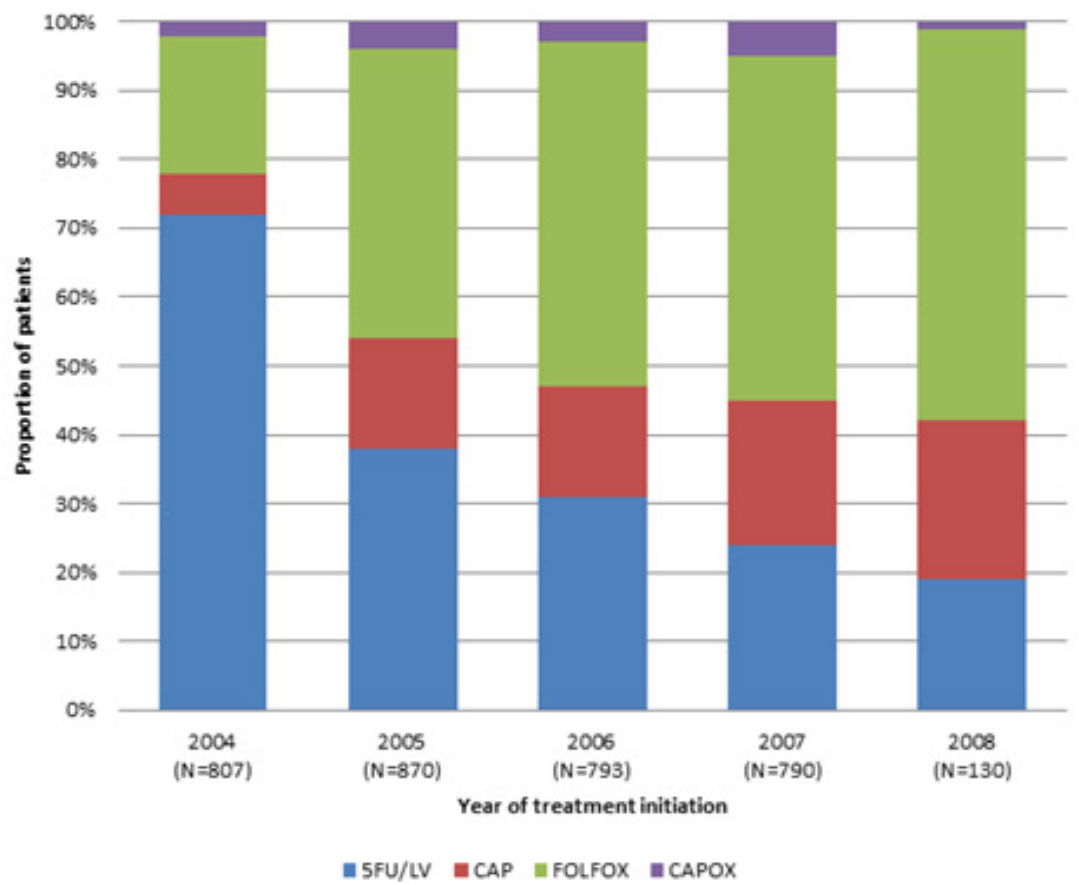

Figure 1. Type of treatment by year of intiation

Table 2. Time in days to adjuvant chemotherapy initiation after surgery and duration of therapy

\begin{tabular}{|c|c|c|c|c|c|c|}
\hline Treatment & $\mathbf{N}$ & Mean & SD & Median & Minimum & Maximum \\
\hline \multicolumn{7}{|c|}{ Time to treatment, days ${ }^{\text {a }}$} \\
\hline $5 \mathrm{FU} / \mathrm{LV}$ & 1368 & 46.70 & 16.82 & 44.00 & 1.00 & 92.00 \\
\hline CAP & 507 & 49.45 & 18.08 & 48.00 & 7.00 & 91.00 \\
\hline FOLFOX & 1398 & 46.88 & 16.29 & 44.00 & 1.00 & 92.00 \\
\hline CAPOX & 117 & 46.04 & 17.19 & 43.00 & 17.00 & 87.00 \\
\hline \multicolumn{7}{|c|}{ Duration of treatment, days ${ }^{b}$} \\
\hline $5 \mathrm{FU} / \mathrm{LV}$ & 1367 & 149.11 & 49.61 & 180.00 & 30.00 & 180.00 \\
\hline CAP & 507 & 121.27 & 59.12 & 143.00 & 30.00 & 180.00 \\
\hline FOLFOX & 1398 & 143.76 & 49.09 & 178.00 & 30.00 & 180.00 \\
\hline CAPOX & 117 & 111.25 & 50.96 & 121.00 & 30.00 & 180.00 \\
\hline
\end{tabular}

\footnotetext{
${ }^{a}$ The first chemotherapy claim within 3 months after surgical resection indicated the start of adjuvant therapy. Time to treatment initiation defined as time from "date of surgery" to "date of first chemotherapy claim".

${ }^{\mathrm{b}}$ Duration of treatment defined as time from date of first chemotherapy claim to 30 days following last administration of first-line agent, or to the day prior to second-line treatment initiation or 30 days following last administration of first-line agent if gap in therapy is $>90$ days
}

\subsection{Treatment Complications}

Patients treated with FOLFOX $(82 \%)$ and 5-FU/LV (78\%) experienced a greater proportion of treatment complications (related to health resource use) than those treated with CAP (74\%) or CAPOX (71\%; $\mathrm{p}=0.006)$. The most common complications were anemia (57\%), nausea/vomiting (32\%), diarrhea (14\%), and neutropenia (10\%) across treatment regimens (Table 3). 
Table 3. Incidence of medical resource utilization related treatment complications within 180 days after treatment initiation

\begin{tabular}{|c|c|c|c|c|c|c|c|c|c|}
\hline \multirow{2}{*}{ Adverse Events } & \multicolumn{2}{|c|}{$5-F U / L V(N=1,368)$} & \multicolumn{2}{|c|}{ CAP $(N=507)$} & \multicolumn{2}{|c|}{ FOLFOX $(\mathrm{N}=1,398)$} & \multicolumn{2}{|c|}{ CAPOX $(\mathrm{N}=117)$} & \multirow[b]{2}{*}{$p$-value } \\
\hline & $\mathrm{n}$ & $(\%)$ & $\mathrm{n}$ & $(\%)$ & $\mathrm{n}$ & $(\%)$ & $\mathrm{n}$ & $(\%)$ & \\
\hline None & 295 & 21.6 & 130 & 25.6 & 249 & 17.8 & 34 & 29.1 & 0.0063 \\
\hline Anemia & 827 & 60.5 & 314 & 61.9 & 746 & 53.4 & 59 & 50.4 & $<0.0001$ \\
\hline Nausea/vomiting & 414 & 30.3 & 86 & 17.0 & 637 & 45.6 & 35 & 29.9 & $<0.0001$ \\
\hline Diarrhea & 171 & 12.5 & 74 & 14.6 & 198 & 14.2 & 11 & 9.4 & 0.1680 \\
\hline Neutropenia & 77 & 5.6 & 17 & 3.4 & 265 & 19.0 & 13 & 11.1 & $<0.0001$ \\
\hline Dehydration & 79 & 5.8 & 33 & 6.5 & 111 & 7.9 & & 5.1 & 0.0298 \\
\hline Thrombocytopenia & & 1.2 & 12 & 2.4 & 21 & 1.5 & & & 0.2255 \\
\hline Sepsis & 15 & 1.1 & & & 17 & 1.2 & & & 0.0059 \\
\hline
\end{tabular}

Note: Cells with counts of less than 11 are suppressed in compliance with the National Cancer Institute's data use agreement for small cell sizes

\subsection{Overall Survival}

Follow-up time varied by treatment (data not shown). Median follow-up duration was similar among patients treated with FOLFOX (560 days, interquartile range [IQR]: 287-826), CAP (496 days, IQR: 244-776), and CAPOX (524 days, IQR: 253-898). Median follow-up duration was significantly longer for 5-FU/LV (824.5 days, IQR: 457.5-1165) than for the other regimens. Patterns for mean follow-up duration were similar to those for median follow-up duration.

Although the median survival time was not reached in all four treatment groups (Figure 2); after 3 years of follow-up the proportion of patients surviving were $85.5 \%( \pm 0.39)$ in the 5 -FU/LV cohort, $91.9 \%( \pm 0.32)$ for FOLFOX, $88.4 \%( \pm 0.53)$ for CAP, and $93.2 \%( \pm 1.23)$ for CAPOX.

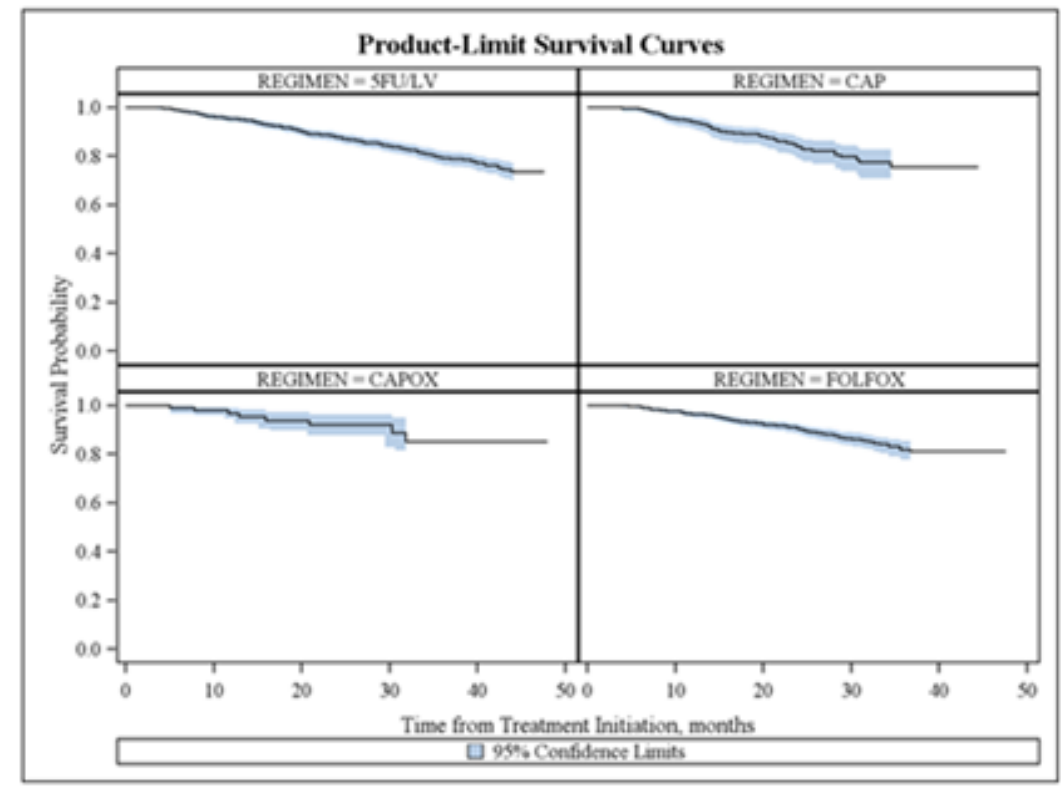

\begin{tabular}{|l|c|c|c|c|c|}
\hline \multirow{2}{*}{ Treatment } & \multicolumn{5}{|c|}{ Number at Risk } \\
\cline { 2 - 6 } & $\mathbf{0}$ months & $\mathbf{1 0}$ months & $\mathbf{2 0}$ months & $\mathbf{3 0}$ months & $\mathbf{4 0}$ months \\
\hline 5-FU/LV & 1368 & 1155 & 910 & 624 & 302 \\
\hline CAP & 507 & 346 & 218 & 76 & \\
\hline FOLFOX & 1398 & 1031 & 650 & 276 & 61 \\
\hline CAPOX & 117 & 81 & 53 & 30 & \\
\hline
\end{tabular}

Note: Cells with counts of less than 11 are suppressed in compliance with the National Cancer Institute's data use agreement for small cell sizes.

Figure 2. Unadjusted overall survival Kaplan-Meier Curve by treatment regimen 
In the multivariate Cox proportional hazards regression analysis, patients treated with FOLFOX had a 28\% lower risk of death than patients treated with 5-FU/LV, and there was no significant difference in hazard of death between patients treated with CAP, CAPOX, or 5-FU/LV (Table 4). Older age, higher number of positive lymph nodes, higher tumor grade, and higher comorbidity score were significant predictors of mortality. In the sensitivity analysis comparing these results to the propensity score-adjusted model, we also found a significant $30 \%$ lower risk of death in patients treated with FOLFOX compared to 5-FU/LV, and no difference between CAP and 5-FU/LV (Table 4). However, the propensity score-adjusted model showed that patients treated with CAPOX had a significantly lower risk of death $(\mathrm{HR}=0.44 ; 95 \% \mathrm{CI}=0.20-0.98)$ than those treated with 5-FU/LV, while there was no significant difference between these two groups in the multivariate Cox proportional hazards model.

Table 4. Overall Survival Analysis

\begin{tabular}{|c|c|c|c|c|c|c|c|}
\hline \multirow[b]{2}{*}{ Treatment } & \multirow[b]{2}{*}{$\mathbf{N}$} & \multicolumn{3}{|c|}{$\begin{array}{l}\text { Multivariate Cox Regression } \\
\text { Reduced Model }^{\mathrm{a}}\end{array}$} & \multicolumn{3}{|c|}{ Propensity Weighted Cox Regression } \\
\hline & & HR & $95 \%$ CI & $p$-value & HR & $95 \%$ CI & $p$-value \\
\hline 5-FU/LV (reference) & 1368 & & & & & & \\
\hline CAP & 507 & 1.261 & $0.95-1.75$ & 0.1311 & 1.167 & $0.88-1.56$ & 0.2925 \\
\hline FOLFOX & 1398 & 0.720 & $0.55-0.94$ & 0.0155 & 0.702 & $0.55-0.90$ & 0.0053 \\
\hline CAPOX & 117 & 0.536 & $0.22-1.31$ & 0.1709 & 0.444 & $0.20-0.98$ & 0.0439 \\
\hline \multicolumn{8}{|l|}{ Age at diagnosis } \\
\hline 66-70 (reference) & 1005 & & & & & & \\
\hline $71-75$ & 1004 & 1.133 & $0.85-1.52$ & 0.4031 & & & \\
\hline $76-80$ & 834 & 1.375 & $1.02-1.85$ & 0.0359 & & & \\
\hline $80>$ & 547 & 1.707 & $1.25-2.33$ & 0.0008 & & & \\
\hline \multicolumn{8}{|l|}{ Positive lymph nodes } \\
\hline 0 (reference) & 854 & & & & & & \\
\hline $1-3$ & 1665 & 1.096 & $0.84-1.43$ & 0.4966 & & & \\
\hline$\geq 4$ & 879 & 2.705 & $2.06-3.55$ & $<.0001$ & & & \\
\hline \multicolumn{8}{|l|}{ Tumor grade } \\
\hline $1-3($ ref) & 2319 & & & & & & \\
\hline $3-4$ & 994 & 1.394 & $1.13-1.72$ & 0.0022 & & & \\
\hline \multicolumn{8}{|l|}{ NCI comorbidity score } \\
\hline 0 (reference) & 854 & & & & & & \\
\hline 1 & 902 & 1.113 & $0.87-1.42$ & 0.3853 & & & \\
\hline$\geq 2$ & 508 & 1.674 & $1.28-2.19$ & 0.0002 & & & \\
\hline \multicolumn{8}{|c|}{ Median income quartiles } \\
\hline 1-Low (reference) & 797 & & & & & & \\
\hline 2 & 869 & 0.946 & $0.71-1.25$ & 0.6994 & & & \\
\hline 3 & 844 & 1.005 & $0.76-1.33$ & 0.9697 & & & \\
\hline 4-High & 873 & 0.737 & $0.54-1.00$ & 0.0477 & & & \\
\hline \multicolumn{8}{|l|}{ Year of diagnosis } \\
\hline 2004 (reference) & 954 & & & & & & \\
\hline 2005 & 878 & 0.744 & $0.58-0.96$ & 0.0243 & & & \\
\hline 2006 & 770 & 0.839 & $0.58-1.20$ & 0.3411 & & & \\
\hline 2007 & 788 & 0.352 & $0.17-0.73$ & 0.0048 & & & \\
\hline
\end{tabular}

HR: hazard ratio; CI: confidence interval

${ }^{a}$ Reduced model by backward elimination. Full model included age, sex, race, positive lymph nodes, tumor grade, comorbidity score, geographic region, income, and year of diagnosis.

${ }^{\mathrm{b}}$ Propensity score weighted for age, sex, race, positive lymph nodes, tumor grade, comorbidity score, geographic region, income, and year of diagnosis. 


\section{Discussion}

This observational study of Medicare beneficiaries with colon cancer, who underwent surgical resection showed a similar survival benefit after adjuvant treatment with $\mathrm{CAP}$ and $5 \mathrm{FU} / \mathrm{LV}$; and a greater overall survival benefit after adjuvant treatment with FOLFOX compared to 5-FU/LV. Despite the hazard ratio being reduced by a similar magnitude in the two statistical models used, the lower risk of death for CAPOX compared to 5FU/LV was only statistically significant in the propensity score-weighted analysis, confirming clinical trial results (Haller et al., 2011). Overall, these results show that the findings from randomized clinical trials (RCTs) in younger patients (Andre et al., 2004; Andre et al., 2009; Haller et al., 2011; Twelves et al., 2011; Twelves et al., 2005) can be generalized to older, frailer patients in real-world settings. Prior research has shown that treatment rates decline dramatically with increasing age (Schrag et al., 2001), and older age is associated with delayed chemotherapy initiation (Hershman et al., 2006), lower rates of chemotherapy completion and less overall use of adjuvant chemotherapy (Bradley, Given, Dahman, \& Fitzgerald, 2008; Dobie et al., 2006). The current findings provide further support that age and comorbidities should not deter the use of guideline-recommended therapies particularly in view of the fact that these disparities are associated with higher mortality (Dobie et al., 2006; Hershman et al., 2006).

Our study inclusion criteria were slightly more stringent than that of a recent SEER-Medicare analysis of adjuvant chemotherapy in Stage III colon cancer (Sanoff et al., 2012). In that study, patients were required to have 6-months of continuous enrollment in Medicare Parts A and B compared to our 12-months of continuous enrollment. Furthermore, we included patients who survived at least 6-months after surgery to account for potential bias related to early deaths post-surgery and initiated chemotherapy within 90-days after surgery to assure that treatment was adjuvant in nature, whereas, the Sanoff et al., study included patients who survived only 30-days after surgery and initiated chemotherapy within 120-days after surgery. In spite of the differences in inclusion criteria between the two studies, the results of the propensity score-weighted analyses were remarkably similar demonstrating the robustness of our statistical approach and findings. We found a significant lower risk of death for FOLFOX compared to 5FU/LV (HR $=0.72 ; 95 \%$ CI: 0.55-0.90) while Sanoff and colleagues reported a significant lower risk of death for FOLFOX compared to non-oxaliplatin regimens, including 5FU/LV with HR= 0.70 ; $95 \%$ CI: $0.60-0.82$.

The patients in this real-world analysis were considerably older, with a median age range of 73-77 compared to those who participated in randomized clinical trials (RCTs) with a median age range of 59-69 (Andre et al., 2004; Andre et al., 2009; Haller et al., 2011; Kuebler et al., 2007; Scheithauer et al., 2003; H. J. Schmoll et al., 2007; Twelves et al., 2011; Twelves et al., 2005). We used a 3-month interval between surgery and initiation of adjuvant chemotherapy which was similar or shorter to the interval used in other observational studies of real-world data (Gross et al., 2007; Sanoff et al., 2012; Schrag et al., 2001; Zuckerman et al., 2009), but slightly longer than the 7 or 8 weeks used in most RCTs. The NCCN recommends starting adjuvant chemotherapy "as soon as medically possible," and analyses of RCT data show an association between treatment benefit and adjuvant chemotherapy initiation within 8 weeks of surgery (Des Guetz, Nicolas, Perret, Morere, \& Uzzan, 2010; National Comprehensive Cancer Network, 2012). Our results show however, that the lessons learned about adjuvant chemotherapy from clinical trials are being broadly applied in routine clinical practice since the majority of patients in this study (70\%) initiated adjuvant chemotherapy within 8 weeks of surgery and $90 \%$ initiated adjuvant therapy within 12 weeks of surgery.

In the current analysis, patients treated with CAP were oldest, and had more comorbidities, compared to other treatment groups. Chemotherapy treatments can be particularly challenging to elderly patients with limited mobility and access to transportation. Even if patients receive their chemotherapy through an ambulatory pump, they still experience discomfort, potential complications (including deep-vein thrombosis), and the need to have a malfunctioning pump replaced (Chu et al., 2012). Elderly patients may prefer oral chemotherapy because of the convenience of administration and concerns over infusion-related problems; provided that such treatments do not compromise treatment efficacy (Borner et al., 2002; Liu, Franssen, Fitch, \& Warner, 1997). Physicians may also perceive the option of an oral chemotherapy, such as CAP, to be of value to elderly patients and may feel that the short remaining natural life expectancy of these patients outweigh the potential benefits of more aggressive treatment (Castiglione, Gelber, \& Goldhirsch, 1990).

Our findings of treatment complications requiring medical resource use confirms results of prior RCTs showing lower AE rates with CAP than 5-FU/LV (Scheithauer et al., 2003; Twelves et al., 2011; Twelves et al., 2005) and higher rates of severe AE with FOLFOX than 5-FU/LV (Andre et al., 2004; Andre et al., 2009; Kuebler et al., 2007). However, in the RCT by Schmoll and colleagues, approximately $20 \%$ of CAPOX patients had grade 3 or 4 diarrhea and $60 \%$ had diarrhea of any severity, whereas only $9 \%$ of CAPOX patients in our study had diarrhea 
(Schmoll et al., 2007). One reason for this difference may be the relatively small number of CAPOX patients in our study, rendering estimates of complication rates unstable. Furthermore, physicians sometimes administer lower chemotherapy doses in elderly patients to increase the tolerability of treatment (Field et al., 2008; Twelves et al., 2005). RCT patients are also monitored closely for the occurrence of AEs; but in this analysis only more serious treatment complications are likely to have been reported given that patients were seen by a physician or received treatment that resulted in a medical claim for payment.

The study has several strengths, including the potentially large sample size from a population-based registry with a wide geographic representation of the United States. The SEER-Medicare dataset includes inpatient and outpatient data, comprehensive information about covered services, all claims regardless of residence or care out of area, and longitudinal data with claims for services from the time a person is eligible for Medicare until their death. Unlike clinical trials, this observational study provides insight into the determinants and effectiveness of adjuvant chemotherapy treatment in actual use in the general population.

Although SEER-Medicare data are a valuable resource for understanding treatment patterns, these data have some limitations. In particular, Medicare does not cover all relevant medical services. As a result, if patients in our study received care not paid for by the Medicare program, the dataset would not have captured this information. No information is available on performance status or lifestyle factors, which affect clinicians' initial decisions to treat. All of the patients in this study use Medicare's fee-for-service (FFS) system, and Medicare HMO patients were excluded because claims are not collected for those patients. Prior research shows that Medicare HMO enrollees with colon cancer had better OS than FFS plan members (Kirsner et al., 2006; Merrill et al., 1999). HMO enrollees tend to be healthier than FFS enrollees and these mortality differences may also be due to higher use of screening and preventive services among HMO patients.

\section{Conclusion}

In conclusion, elderly colon cancer patients treated in real-world settings experienced similar benefits from adjuvant chemotherapies as younger patients in clinical trial settings, and imply that age and comorbidities should not discourage the use of guideline-recommended therapies. Further research is required to evaluate possible interactions of performance status on patterns and outcomes of care, and incidence of treatment-related complications. In addition, a comparison of the treatment patterns, treatment complications and survival outcomes of patients enrolled in HMOs compared with those in FFS plans is warranted.

\section{Acknowledgements}

Funding for this study was provided by Genentech, Inc. We also acknowledge the efforts of the Applied Research Program, NCI (Bethesda, MD); the Office of Information Services and the Office of Strategic Planning, Health Care Financing Administration (Baltimore, MD); Information Management Services, Inc. (Silver Spring, MD); and the SEER program tumor registries in the creation of the SEER-Medicare database. The interpretation and reporting of these data are the sole responsibility of the authors. Deborah Berlyne, Ph.D., helped prepare this manuscript.

\section{References}

Andre, T., Boni, C., Mounedji-Boudiaf, L., Navarro, M., Tabernero, J., Hickish, T., ... Multicenter International Study of Oxaliplatin/5-Fluorouracil/Leucovorin in the Adjuvant Treatment of Colon Cancer Investigators. (2004). Oxaliplatin, fluorouracil, and leucovorin as adjuvant treatment for colon cancer. $N$ Engl J Med, 350(23), 2343-2351. http://dx.doi.org/10.1056/NEJMoa032709

Andre, T., Boni, C., Navarro, M., Tabernero, J., Hickish, T., Topham, C., ... de Gramont, A. (2009). Improved overall survival with oxaliplatin, fluorouracil, and leucovorin as adjuvant treatment in stage II or III colon cancer in the MOSAIC trial. J Clin Oncol, 27(19), 3109-3116. http://dx.doi.org/10.1200/JCO.2008.20.6771

Borner, M. M., Schoffski, P., de Wit, R., Caponigro, F., Comella, G., Sulkes, A., ... Fumoleau, P. (2002). Patient preference and pharmacokinetics of oral modulated UFT versus intravenous fluorouracil and leucovorin: a randomised crossover trial in advanced colorectal cancer. Eur J Cancer, 38(3), 349-358.

Bradley, C. J., Given, C. W., Dahman, B., \& Fitzgerald, T. L. (2008). Adjuvant chemotherapy after resection in elderly Medicare and Medicaid patients with colon cancer. Arch Intern Med, 168(5), 521-529. http://dx.doi.org/10.1001/archinternmed.2007.82

Castiglione, M., Gelber, R. D., \& Goldhirsch, A. (1990). Adjuvant systemic therapy for breast cancer in the elderly: competing causes of mortality. International Breast Cancer Study Group. J Clin Oncol, 8(3), 519-526. 
Chu, E., Haller, D. G., Cartwright, T. H., Twelves, C., McKenna, E., Scotto, N., ... Schmoll, H.-J. (2012). Epidemiology and natural history of central venous access device (CVAD) use and infusion pump performance among patients (pts) treated for metastatic colorectal cancer ( $m C R C)$ : Analysis from the NO16966 trial. Paper presented at the 2012 Gastrointestinal Cancers Symposium, San Francisco, CA. http://www.asco.org/ASCOv2/Meetings/Abstracts?\&vmview=abst_detail_view\&confID=115\&abstractID= 88795

Des Guetz, G., Nicolas, P., Perret, G. Y., Morere, J. F., \& Uzzan, B. (2010). Does delaying adjuvant chemotherapy after curative surgery for colorectal cancer impair survival? A meta-analysis. European journal of cancer, 46(6), 1049-1055. http://dx.doi.org/10.1016/j.ejca.2010.01.020

Dobie, S. A., Baldwin, L. M., Dominitz, J. A., Matthews, B., Billingsley, K., \& Barlow, W. (2006). Completion of therapy by Medicare patients with stage III colon cancer. J Natl Cancer Inst, 98(9), 610-619. http://dx.doi.org/10.1093/jnci/djj159

Field, K. M., Kosmider, S., Jefford, M., Michael, M., Jennens, R., Green, M., \& Gibbs, P. (2008). Chemotherapy dosing strategies in the obese, elderly, and thin patient: results of a nationwide survey. J Oncol Pract, 4(3), 108-113. http://dx.doi.org/10.1200/jop.0832001

Greenland, S. (1998). Modern Epidemiology (2nd Edition ed.). Philadelphia: Lippincott-Raven Publishers.

Gross, C. P., McAvay, G. J., Guo, Z., \& Tinetti, M. E. (2007). The impact of chronic illnesses on the use and effectiveness of adjuvant chemotherapy for colon cancer. Cancer, 109(12), 2410-2419. http://dx.doi.org/10.1002/cncr.22726

Haller, D. G., Tabernero, J., Maroun, J., de Braud, F., Price, T., Van Cutsem, E., ... Schmoll, H. J. (2011). Capecitabine plus oxaliplatin compared with fluorouracil and folinic acid as adjuvant therapy for stage III colon cancer. J Clin Oncol, 29(11), 1465-1471. http://dx.doi.org/10.1200/JCO.2010.33.6297

Hershman, D., Hall, M. J., Wang, X., Jacobson, J. S., McBride, R., Grann, V. R., \& Neugut, A. I. (2006). Timing of adjuvant chemotherapy initiation after surgery for stage III colon cancer. Cancer, 107(11), 2581-2588. http://dx.doi.org/10.1002/cncr.22316

International Multicentre Pooled Analysis of B2 Colon Cancer Trials (IMPACT B2) Investigators (1995). Efficacy of adjuvant fluorouracil and folinic acid in colon cancer. Lancet, 345(8955), 939-944.

Jessup, J. M., Stewart, A., Greene, F. L., \& Minsky, B. D. (2005). Adjuvant chemotherapy for stage III colon cancer: implications of race/ethnicity, age, and differentiation. JAMA, 294(21), 2703-2711.

Kahn, K. L., Adams, J. L., Weeks, J. C., Chrischilles, E. A., Schrag, D., Ayanian, J. Z., ... Fletcher, R. H. (2010). Adjuvant chemotherapy use and adverse events among older patients with stage III colon cancer. JAMA, 303(11), 1037-1045. http://dx.doi.org/10.1001/jama.2010.272

Kirsner, R. S., Ma, F., Fleming, L., Federman, D. G., Trapido, E., Duncan, R., \& Wilkinson, J. D. (2006). The effect of medicare health care delivery systems on survival for patients with breast and colorectal cancer. Cancer Epidemiol Biomarkers Prev, 15(4), 769-773. http://dx.doi.org/10.1158/1055-9965.EPI-05-0838

Klabunde, C. N., Legler, J. M., Warren, J. L., Baldwin, L. M., \& Schrag, D. (2007). A refined comorbidity measurement algorithm for claims-based studies of breast, prostate, colorectal, and lung cancer patients. Ann Epidemiol, 17(8), 584-590. http://dx.doi.org/10.1016/j.annepidem.2007.03.011

Kuebler, J. P., Wieand, H. S., O'Connell, M. J., Smith, R. E., Colangelo, L. H., Yothers, G., ... Wolmark, N. (2007). Oxaliplatin combined with weekly bolus fluorouracil and leucovorin as surgical adjuvant chemotherapy for stage II and III colon cancer: results from NSABP C-07. J Clin Oncol, 25(16), 2198-2204. http://dx.doi.org/10.1200/JCO.2006.08.2974

Kurth, T., Walker, A. M., Glynn, R. J., Chan, K. A., Gaziano, J. M., Berger, K., \& Robins, J. M. (2006). Results of multivariable logistic regression, propensity matching, propensity adjustment, and propensity-based weighting under conditions of nonuniform effect. Am $J$ Epidemiol, 163(3), 262-270. http://dx.doi.org/10.1093/aje/kwj047

Liu, G., Franssen, E., Fitch, M. I., \& Warner, E. (1997). Patient preferences for oral versus intravenous palliative chemotherapy. J Clin Oncol, 15(1), 110-115.

Merrill, R. M., Brown, M. L., Potosky, A. L., Riley, G., Taplin, S. H., Barlow, W., \& Fireman, B. H. (1999). Survival and treatment for colorectal cancer Medicare patients in two group/staff health maintenance organizations and the fee-for-service setting. Medical care research and review : MCRR, 56(2), 177-196. 
Moertel, C. G., Fleming, T. R., Macdonald, J. S., Haller, D. G., Laurie, J. A., Tangen, C. M., ... Mailliard, J. A. (1995). Fluorouracil plus levamisole as effective adjuvant therapy after resection of stage III colon carcinoma: a final report. Ann Intern Med, 122(5), 321-326.

National Comprehensive Cancer Network. (2012, January 17, 2012). NCC Clinical Practice Guidelines in Oncology (NCCN Guidelines(r): Colon Cancer. Version 3.2012. Retrieved February 24, 2012, from http://www.nccn.org/professionals/physician_gls/f_guidelines.asp

NCCN. (National Comprehensive Cancer Network). (2012). Clinical Practice Guidelines in Oncology. $<$ http://www.nccn.org/professionals/physician_gls/pdf/colon.pdf> V.3.2012.

O'Grady, M. A., Slater, E., Sigurdson, E. R., Meropol, N. J., Weinstein, A., Lusch, C. J., ... Cohen, S. J. (2011). Assessing compliance with national comprehensive cancer network guidelines for elderly patients with stage III colon cancer: the Fox Chase Cancer Center Partners' initiative. Clin Colorectal Cancer, 10(2), 113-116. http://dx.doi.org/10.1016/j.clcc.2011.03.007

Potosky, A. L., Riley, G. F., Lubitz, J. D., Mentnech, R. M., \& Kessler, L. G. (1993). Potential for cancer related health services research using a linked Medicare-tumor registry database. Med Care, 31(8), 732-748.

Sanoff, H. K., Carpenter, W. R., Martin, C. F., Sargent, D. J., Meyerhardt, J. A., Sturmer, T., ... Schrag, D. (2012). Comparative effectiveness of oxaliplatin vs non-oxaliplatin-containing adjuvant chemotherapy for stage III colon cancer. Journal of the National Cancer Institute, 104(3), 211-227. http://dx.doi.org/10.1093/jnci/djr524

Scheithauer, W., McKendrick, J., Begbie, S., Borner, M., Burns, W. I., Burris, H. A., ... Twelves, C. (2003). Oral capecitabine as an alternative to i.v. 5-fluorouracil-based adjuvant therapy for colon cancer: safety results of a randomized, phase III trial. Annals of oncology: official journal of the European Society for Medical Oncology / ESMO, 14(12), 1735-1743.

Schmoll, H.-J., Tabernero, J., Maroun, J., de Braud, F., Price, T. J., Van Cutsem, E., ... Haller, D. G. (2012). Capecitabine plus oxaliplatin (XELOX) versus bolus 5-fluorouracil/leucovorin (5-FU/LV) as adjuvant therapy for stage III colon cancer: Survival follow-up of study NO16968 (XELOXA). Paper presented at the 2012 Gastrointestinal Cancers Symposium, San Francisco, CA. http://www.asco.org/ASCOv2/Meetings/Abstracts?\&vmview=abst_detail_view\&confID=115\&abstractID= 88795

Schmoll, H. J., Cartwright, T., Tabernero, J., Nowacki, M. P., Figer, A., Maroun, J., ... Haller, D. G. (2007). Phase III trial of capecitabine plus oxaliplatin as adjuvant therapy for stage III colon cancer: a planned safety analysis in 1,864 patients. $J$ Clin Oncol, 25(1), 102-109. doi: 10.1200/JCO.2006.08.1075

Schrag, D., Cramer, L. D., Bach, P. B., \& Begg, C. B. (2001). Age and adjuvant chemotherapy use after surgery for stage III colon cancer. J Natl Cancer Inst, 93(11), 850-857.

Schrag, D., Gelfand, S. E., Bach, P. B., Guillem, J., Minsky, B. D., \& Begg, C. B. (2001). Who gets adjuvant treatment for stage II and III rectal cancer? Insight from surveillance, epidemiology, and end results--Medicare. J Clin Oncol, 19(17), 3712-3718.

Sundararajan, V., Mitra, N., Jacobson, J. S., Grann, V. R., Heitjan, D. F., \& Neugut, A. I. (2002). Survival associated with 5-fluorouracil-based adjuvant chemotherapy among elderly patients with node-positive colon cancer. Ann Intern Med, 136(5), 349-357.

Twelves, C., Scheithauer, W., McKendrick, J., Seitz, J. F., Van Hazel, G., Wong, A., ... Cassidy, J. (2011). Capecitabine versus 5-fluorouracil/folinic acid as adjuvant therapy for stage III colon cancer: final results from the X-ACT trial with analysis by age and preliminary evidence of a pharmacodynamic marker of efficacy. Annals of oncology : official journal of the European Society for Medical Oncology / ESMO.

Twelves, C., Wong, A., Nowacki, M. P., Abt, M., Burris, H., 3rd, Carrato, A., ... Scheithauer, W. (2005). Capecitabine as adjuvant treatment for stage III colon cancer. $N$ Engl $J$ Med, 352(26), 2696-2704. http://dx.doi.org/10.1056/NEJMoa043116

Warren, J. L., Harlan, L. C., Fahey, A., Virnig, B. A., Freeman, J. L., Klabunde, C. N., ... Knopf, K. B. (2002). Utility of the SEER-Medicare data to identify chemotherapy use. Med Care, 40(8 Suppl), IV-55-61. http://dx.doi.org/10.1097/01.MLR.0000020944.17670.D7

Warren, J. L., Klabunde, C. N., Schrag, D., Bach, P. B., \& Riley, G. F. (2002). Overview of the SEER-Medicare data: content, research applications, and generalizability to the United States elderly population. Med Care, 40(8 Suppl), IV-3-18. http://dx.doi.org/10.1097/01.MLR.0000020942.47004.03 
Wolmark, N., Rockette, H., Fisher, B., Wickerham, D. L., Redmond, C., Fisher, E. R., ... Petrelli, N. J. (1993). The benefit of leucovorin-modulated fluorouracil as postoperative adjuvant therapy for primary colon cancer: results from National Surgical Adjuvant Breast and Bowel Project protocol C-03. J Clin Oncol, 11(10), 1879-1887.

Wolpin, B. M., Meyerhardt, J. A., Mamon, H. J., \& Mayer, R. J. (2007). Adjuvant treatment of colorectal cancer. CA Cancer J Clin, 57(3), 168-185.

Zuckerman, I. H., Rapp, T., Onukwugha, E., Davidoff, A., Choti, M. A., Gardner, J., ... Mullins, C. D. (2009). Effect of age on survival benefit of adjuvant chemotherapy in elderly patients with Stage III colon cancer. $J$ Am Geriatr Soc, 57(8), 1403-1410. http://dx.doi.org/10.1111/j.1532-5415.2009.02355.x 